\title{
An effect of manjistha khadir choorna orally and dermocare oil locally in the management of vicharchika w. s. r. to eczema -a case study.
}

\author{
Subhash B. Jamdhade ${ }^{1}$, Monica W. Chavan ${ }^{2}$, S. K. Jaiswal ${ }^{3}$, Pradnya, S. Jamdhade \\ 1. Associate Professor \\ 2. PG scholar \\ 3. HOD/Professor \\ 4. Assistant Professor \\ P.G. Dept of Kayachikitsa, D.M.M Ayurved College, Yavatmal, M. S. (India) \\ *Corresponding author: monica.chavan94@gmail.com
}

\begin{abstract}
:
In Ayurveda, majority of skin diseases have been mentioned under the umbrella refractory to treatment But Ayurveda is the repository of skin remedies which are therapeutically safe and effectiveness. In of Kushthaand in other words, it can/be present case study is successful listed as "Ayurvedic dermatology" aytrvedic management of case of Ayurveda is science of life. Ayurveda is not only curative but also preventive science of life. In ayurveda the word Kushta is broad term which cover almost all skin disorder. Vicharchika is a type of kshudrakushta often encountered by Ayurvedic dermatologists characterized with symptoms namely Kandu (Itching) Srava ( discharge) Pidika (vesicles) and Shayava varna (Discoloration) Rajyo (marked lining due to thickness of lesions) Vicharchika is often correlated to eczema based on the clinical presentations. No satisfactory treatment is available in contemporary medical practice expect antihistamines and topical steroids. Main line of treatment for vicharchika in Ayurved is shodhsna and shaman oushadhi. In spite of presence of antibiotic, antihistamines, steroids etc, the skin disorder remains report of a 45 years male having with complaint of padbhagi twak vaivaranyata kandu pidika ruja rajyo rukshta strava. He was treated with ayurvedic drugs. A remarkable improvement in comdition was observed.

Keywords: kushta, eczema, shaman shoudhan oushadhi, vicharchika

\section{Introduction}

Ayurveda is a life science. Ayurveda is not only curative but also preventative science of life . In Ayurveda, all skin disorders describes under the heading of Kushta. Skin is the one of the sense organ in the body. largest and important organ and outermost covering of the body which acts as a protective barrier. But very often, it is exposed to many entities which can damage it and cause
\end{abstract}


many of the skin disorder. About 10$20 \%$ of the general practice includes the patients suffering from skin disorder and Eczema accounts for a very large proportion of all the skin diseases. In Ayurveda, The word Kushta is broad term, which covers almost all skin disorders.

"kushnatee iti kushtham"
(Ma.Ni./Kushta./Tika 1)

Viz. $\quad 1 . \quad$ Mahakushtha, 2. Kshudrakushtha,

Vicharchika being one among kshudrakushtha, is also a Rasa, Rakta and Mansa dhatu pradoshaj vikara.

Vicharchika Nirukti: The word vicharchika is derived from the root word "Charcha" with "Vi" upasarg meaning a form Coetaneous Eruption with itch and scab.

Paribhasha: The term Vicharchika is derived as one of the variety/of Astadasha Kshudrakushta, in which the skin develops Shyavvarnata, Pidaka, associated with srav and kandu. According to Acharya vicharchika is kaphapradhan vyadhi and symptoms of vicharchika are kandu (Itching), shyava varna (blackish discolouration), pidika (carbuncles), bahu strava (discharge), ruja (pain), rajyo (marked lining due to thickness of lesion ), rukshata (dryness). Also Many Acharya defined as various dosha responsible for Vicharchika vyadhi and defined vicharchika is kaphapradhan, pittapradhan, Kaphavatprdhan Disease. Vicharchika is described in many of Ayurved text. विचर्चिका-

१)सकण्डु पिडका श्यावा बहुस्त्रावा
विचर्चिका||

‘च.चि.७/२६,यो.र/भा.प्र.५४/२७
2)कफप्रायाविचर्चिका॥च.चि.७/३०

3)राज्योअतिकण्ड्वर्तिरुज:

सरुक्षाभवन्तिगात्रेषुविचर्चिकायाम्॥..सु.

नि. $९ / १ ३$

4)सकण्डुपिटीकाश्यावालसीकाढ्याविचर्चि का॥ अ.ह्.नि $१ ४ / १ ८$

5)श्यामलोहितव्रणवेदनास्त्रावपाकवतीवि चर्चि॥..का.सं Pg.No.116

6) पित्तेनोदुम्बरंप्रोक्तंकफान्मण्डलचर्चिके

\section{॥ शा.सं७/८}

All the clinical features of vicharchika can be very well correlate to clinical features of Eczema, which is distinctive pattern of inflammatory response of the skin. Eczema is broad term used for many types of skin inflammations, It is the skin disorder also called as Dermatitis. It is a pattern of inflammatory response of the skin which is the resultant of delayed type of hypersensitivity mediated $\mathrm{T}$ lymphocytes in the skin. It clinically manifest by pruritus, eythema, oedema, Papules and Vesicles and oozing in acute stage wheras itching, scaling, dryness and lichenification occur in chronic stage.

Appearance carries a lot of weight in modern world. Patients are very much concern about grooming and the way they present themselves before other. Both men and women want a looking attractive and fabulous skin right till old age. Any blemish on skin causes lot of psychological stress, physical uneasiness. In spite of presence of antibiotic, antihistamines, steroids etc, the skin disorder remains refractory to treatment But Ayurveda is the repository of skin remedies which are therapeutically safe and effectiveness. 
The prevalence of skin disease in the general population has varied from $7.86 \%$ to $11.16 \%$ in various studies. According to JMGIMS (journal of mahatma Gandhi institute of medical science.),Eczema is the most common dermatitis in the last 10 years.

\section{Method:- A case report}

A $45 \mathrm{yr}$ male pt. came to opd of kayachikitsa department of $\mathrm{L} \mathrm{K}$ Ayurved Hospital yavatmal with chief c/o

1) Padbhagi Twak vaivaranyata (changes in skin coloration) since 3 yr.

2) $k a n d u$ (Itching) since $3 \mathrm{yr}$

3) pidika (eruption), since $3 \mathrm{yr}$

4) Shyav varna (blackish discoloration) since $3 \mathrm{yr}$

5) Strava (discharge), on and off

6) Ruja (pain), since $3 \mathrm{yr}$

7) Rajyo (marked lining due to thickness of lesion ), since $3 \mathrm{yr}$

8) Rukshata (dryness), since $3 \mathrm{yr}$

Pt. having above complaints si $3 \mathrm{yr}$

Past history:- No H/O DM/HTN/ Any major illness.

History of present illness :- pt. is normal before $3 \mathrm{yr}$ ago then pt. has been sufferd from above symptoms. Pt. was treated by local doctor many times but after stop medication pt. symptoms was aggravated then pt. came to L.K. Ayurved Hospital yavatmal for Ayurvedic treatment and management.

\section{Rugna parikshan:-}

- Nadi:-68/min

- Mal: Asamyak

- Mutra: samyak

- Jivha: alpa saam

- Shabda : prakrut

- Sparsha : prakrut

- Druk : praktrut

- Aakruti:-Madhyam

- Nidra:-prakrut

- BP:- 140/80 $\mathrm{mmHg}$

- Temp:- Afebrile

\section{Material and methods}

Method:-

\section{A Case Study}

Centre: P.G. Dep. Of Kayachikitsa

TABLE NO.1

\begin{tabular}{|l|l|l|l|l|}
\hline Sr no & \multicolumn{1}{|c|}{ Dravya } & Dose & Duration & Anupan \\
\hline 1 & $\begin{array}{l}\text { Manjistha khadir } \\
\text { choorna }\end{array}$ & $3 \mathrm{gm}$ & Twice a day & $\begin{array}{l}\text { Luke warm } \\
\text { water }\end{array}$ \\
\hline 2 & Dermacare oil & $\begin{array}{l}\text { Local } \\
\text { application }\end{array}$ & Twice a day & \\
\hline
\end{tabular}


TABLE NO. 2

1. MANJISHTA KHADIR CHOORNA:

\begin{tabular}{|c|l|l|l|l|l|l|}
\hline $\begin{array}{c}\text { Sr. } \\
\text { no. }\end{array}$ & $\begin{array}{l}\text { Sankrit } \\
\text { name }\end{array}$ & $\begin{array}{l}\text { Botanical } \\
\text { name }\end{array}$ & Ras & Virya & Vipak & Karmukta \\
\hline 1 & Manjistha & $\begin{array}{l}\text { Rubia } \\
\text { cordiafolia }\end{array}$ & Kashay & Ushna & Katu & $\begin{array}{l}\text { Kapha } \\
\text { Pittashamak }\end{array}$ \\
\hline 2 & Khadir & $\begin{array}{l}\text { Acasia } \\
\text { catechu }\end{array}$ & $\begin{array}{l}\text { Tikta } \\
\text { kashay }\end{array}$ & Shita & Katu & $\begin{array}{l}\text { Kaphapitta } \\
\text { Shamak }\end{array}$ \\
\hline
\end{tabular}

TABLE NO 32 DERMOCARE OIL

\begin{tabular}{|c|c|c|c|c|c|c|}
\hline Sr. & $\begin{array}{l}\text { Sanskrit } \\
\text { name }\end{array}$ & Latinname & ras & virya & $\begin{array}{l}\text { vipa } \\
\mathrm{k}\end{array}$ & karmukta \\
\hline 1 & Kalamari & Pipernigam & katu & $\begin{array}{l}\text { ushn } \\
a\end{array}$ & katu & lekhan \\
\hline 2 & Ark & $\begin{array}{l}\text { Calotropis } \\
\text { gigantea }\end{array}$ & katu & $\begin{array}{l}\text { ushn } \\
a\end{array}$ & katu & $\begin{array}{l}\text { kaphavatsha } \\
\text { mak }\end{array}$ \\
\hline 3 & Devdar & cedrusdeodara & $\begin{array}{l}\text { Tikta } \\
\text { katu }\end{array}$ & $\begin{array}{l}\text { ushn } \\
a\end{array}$ & katu & kushtanng \\
\hline 4 & Haridra & Curcuma longa & & $\begin{array}{l}\text { ushn } \\
a\end{array}$ & katu & $\begin{array}{l}\text { Kaphavat } \\
\text { shamak }\end{array}$ \\
\hline 5 & $\begin{array}{l}\text { Daruhari } \\
\text { dra }\end{array}$ & Berberis aristafa & Relkital & $\begin{array}{l}\text { ushn } \\
a\end{array}$ & katu & $\begin{array}{l}\text { kaphapittaha } \\
r\end{array}$ \\
\hline 6 & Kath & Sassurea lappa & $\begin{array}{l}\text { hay } \\
\text { hiktakas }\end{array}$ & shita & katu & $\begin{array}{l}\text { Kapha } \\
\text { pittashamak }\end{array}$ \\
\hline 7 & $\begin{array}{l}\text { Rraktachand } \\
\text { an }\end{array}$ & pterocarcaus & tikta & shita & katu & $\begin{array}{l}\text { kaphapittash } \\
\text { amak }\end{array}$ \\
\hline 8 & Indravarun & $\begin{array}{l}\text { Citrullus } \\
\text { colocynthis }\end{array}$ & tikta & $\begin{array}{l}\text { ushn } \\
a\end{array}$ & katu & $\begin{array}{l}\text { kaphapittagh } \\
\text { na }\end{array}$ \\
\hline 9 & Kaner & Nerium indicum & katu & $\begin{array}{l}\text { usha } \\
n a\end{array}$ & katu & $\begin{array}{l}\text { kaphavatsha } \\
\text { mak }\end{array}$ \\
\hline $\begin{array}{l}1 \\
0\end{array}$ & Chitrakmool & $\begin{array}{l}\text { Plumbago } \\
\text { zeylanica }\end{array}$ & katu & $\begin{array}{l}\text { ushn } \\
a\end{array}$ & katu & $\begin{array}{l}\text { vatkaphasha } \\
\text { mak }\end{array}$ \\
\hline $\begin{array}{l}1 \\
1\end{array}$ & Vavdinga & Embelica ribes & katu & $\begin{array}{l}\text { ushn } \\
a\end{array}$ & katu & $\begin{array}{l}\text { vatakaphasha } \\
\text { mak }\end{array}$ \\
\hline $\begin{array}{l}1 \\
2\end{array}$ & $\begin{array}{l}\text { Chakramard } \\
\text { a }\end{array}$ & Cassia tora & katu & shita & katu & $\begin{array}{l}\text { kaphavatagh } \\
\text { ana }\end{array}$ \\
\hline $\begin{array}{l}1 \\
3\end{array}$ & $\begin{array}{l}\text { Shirishch } \\
\text { hal }\end{array}$ & Albizia lebbeck & kashay & $\begin{array}{l}\text { ushn } \\
a\end{array}$ & katu & $\begin{array}{l}\text { tridoshshama } \\
k\end{array}$ \\
\hline $\begin{array}{l}1 \\
4\end{array}$ & Kutaj & $\begin{array}{l}\text { Holarrhena } \\
\text { antidysenterica }\end{array}$ & $\begin{array}{l}\text { tkiktaks } \\
\text { hay }\end{array}$ & shita & katu & $\begin{array}{l}\text { kaphapittash } \\
\text { amak }\end{array}$ \\
\hline 1 & Neemchh & Azadirachta & tikta & shita & katu & kaphapittash \\
\hline
\end{tabular}




\begin{tabular}{|c|c|c|c|c|c|c|}
\hline 5 & al & indica & & & & amak \\
\hline $\begin{array}{l}1 \\
6\end{array}$ & Galo & $\begin{array}{l}\text { Tinosporacordi } \\
\text { folia }\end{array}$ & tikta & $\begin{array}{l}\text { ushn } \\
a\end{array}$ & katu & $\begin{array}{l}\text { kaphapittash } \\
\text { amak }\end{array}$ \\
\hline $\begin{array}{l}1 \\
7\end{array}$ & Amaltas & Cassia fistula & amla & $\begin{array}{l}\text { ushn } \\
a\end{array}$ & $\begin{array}{l}a m l \\
a\end{array}$ & $\begin{array}{l}\text { kaphavatsha } \\
\text { mak }\end{array}$ \\
\hline $\begin{array}{l}1 \\
8\end{array}$ & $\begin{array}{l}\text { Karanbee } \\
\text { j }\end{array}$ & $\begin{array}{l}\text { Pongamia } \\
\text { glabra }\end{array}$ & tikta & $\begin{array}{l}\text { ushn } \\
a\end{array}$ & katu & kaphavatshak \\
\hline $\begin{array}{l}1 \\
9\end{array}$ & $\begin{array}{l}\text { Nagarmo } \\
\text { tha }\end{array}$ & $\begin{array}{l}\text { Cyprus } \\
\text { rotundus }\end{array}$ & $\begin{array}{l}\text { katu } \\
\text { tikta } \\
\text { kashay }\end{array}$ & shitat & katu & pittaghan \\
\hline $\begin{array}{l}2 \\
0\end{array}$ & $\begin{array}{l}\text { Kherchha } \\
1\end{array}$ & Acacia catechu & $\begin{array}{l}\text { tikta } \\
\text { kashay }\end{array}$ & shita & katu & $\begin{array}{l}\text { kaphavatagh } \\
\text { ana }\end{array}$ \\
\hline $\begin{array}{l}2 \\
1\end{array}$ & Vaj & acoruscalamus & katu & shita & katu & $\begin{array}{l}\text { kaphavatagh } \\
\text { na }\end{array}$ \\
\hline
\end{tabular}

showing changes in affected area before chikitsa and after chikitsa Treatment

Before treatment:-

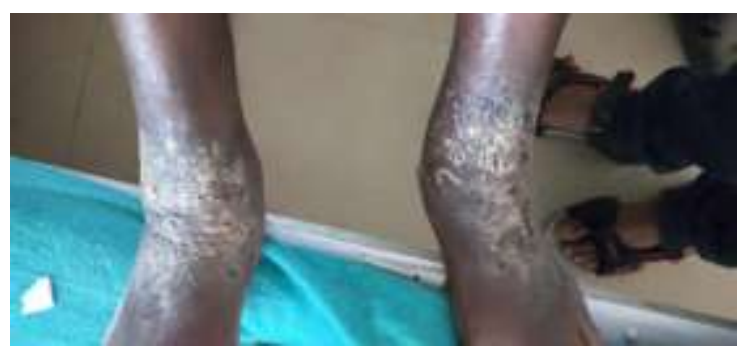

During treatment :-1.

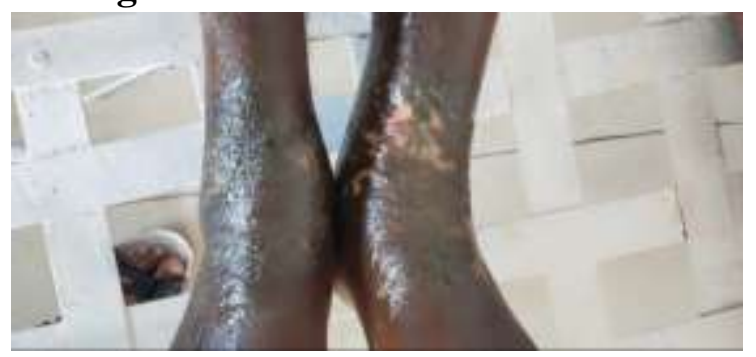

During treatment 2.

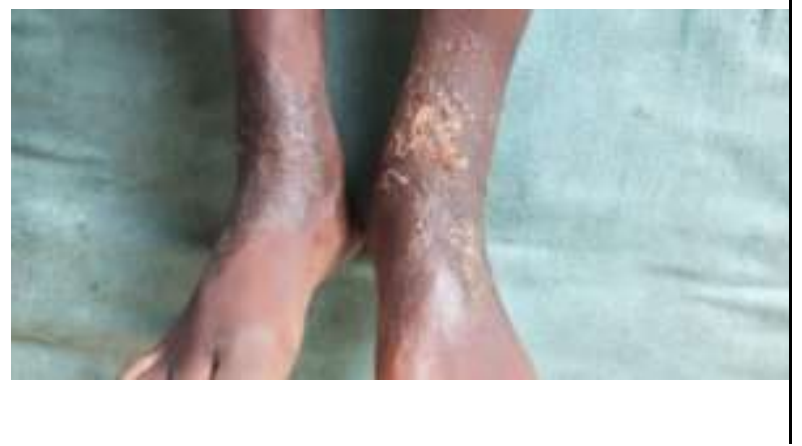

After treatment:-

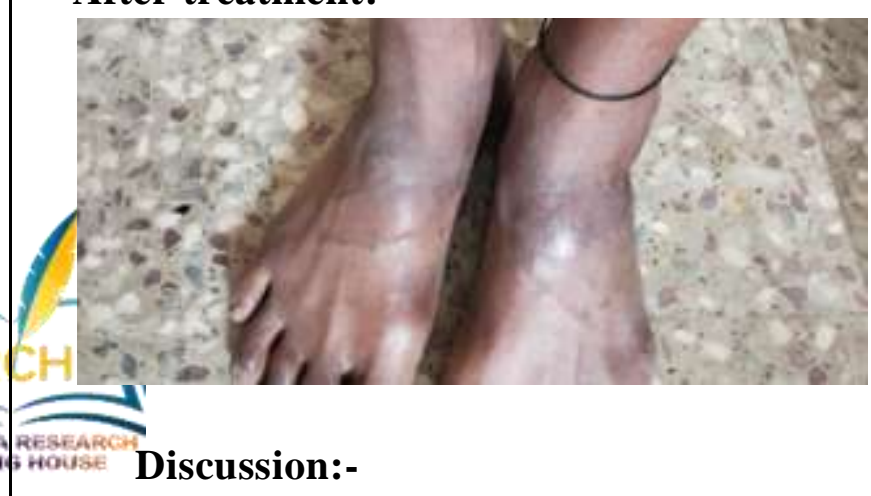

Hetu:-

1) Aahar:- Dadhi sevan, Mansa sevan,

Lavan-amla-katu padarth sevan

Madhyapan, Virudha aahar, Vidahi annapan

Vihar:-Atishram, Aatapsevan, Atikrodh, Panchkarma Abhav, Diwaswap

Samprapti Ghatak:-

1)Dosha:- vat + kapha

2)Dushya:- Rasa, Rakta,Mansa

3)Strotas:- Rasavaha strotas

Raktavaha strotas

Mansavah strotas

4) Adhisthan:-Rasa,Rakta,Mansa

5)Udbhavsthan:- Twaka

6)Vyaktisthan:- Twaka 


\section{CONCLUSION}

In this study the drug used orally formulation of majistha choorna most helpful in all types of skin disorders an imbalanced pitta dosha vitiates the blood and impairs its normal functioning. This causes skin problems like redness manjistha helps to purify the blood and treats all type of skin disorders. this is due to its pitta balancing and rakta shodhak (blood purifier properties). Khadiardi choorna which mostly acts as Kushtaghna, vranaghna, twachyakar, Shothaghna, Raktashudhikar, Balancing trishosha and rasayani. Locally used dermocare oil which also Kushtaghna, vranaghna, twachyakar, Shothaghna, Raktashudhikar. Application of dermocare oil would take care of rukshta (dryness) as it contains til tailam which is snehpaakdrya. Haridra daruharida reduces acts as shodhagana i.e reduces swelling. Chakramarda acts vishaghana decreases kandu (itching) Raktachandan acts as dahaprashmak reduces (burning sensation) Chitrakmula acts as lekhna i.e. it removes pitika (eruptions) nagarmotha and nimba acts as kledaghna decrease kandu (itching).

Dermocare oil fights free radicals that damage cell membrane. Hydrating properties in the oil will relive from itching. Anti-inflammatory and antibacterial infection to relive inflammation. From above case study. It is concluded that Ayurvedic drug formulation of manjishtakhadir choorna orally and dermocare oil locally is very effective to reduce sign and symptoms of vicharchika patient.

\section{REFRENCES:}

1. Yadavji trikamji chaukhamba 1941 charak samhita pub by sanskrit series office, varanasi.

2. Kunjalal kaviraj 1911 sushruta samhita of shushruta vol. 2 pub by author calcutta P Nos 22-29 ,35,42

3. Shastry $s$ \& Upadhyaya y 1975 Madhava nidana $4^{\text {th }}$ edn pub.by choukhamba sanskrit samsthan varanasi .

4. Dravyaguna vigyan vol 1, 2, 3 Priyavat Sharma Pub. by choukhamba sanskrit prakashan.

. Kunte A.M \& Navre, K RS Ashtanga hridya of vagbhata edited paradkar, pub by choukhamba sanskrit samsthan varanasi.
Conflict of Interest: Non

Cite this article:

An effect of manjistha khadir choorna orally and dermocare oil locally in the management of vicharchika w. s. r. to eczema - a case study.

Subhash B Jamdhade, Monica W. Chavan, S. K. Jaiswal, Pradnya S. Jamdhade 